\title{
ANÁLISIS CUANTITATIVO DEL MANTENIMIENTO DE LA REFERENCIA ANAFÓRICA EN NGÄBE (GUAYMÍ)
}

\author{
CUANTITATIVE ANALYSIS ABOUT MAINTAINING THE ANAPHORIC \\ REFERENCE IN NGÄBE (GUAYMÍ)**
}

Haakon Stensrud Krohn*

\section{RESUMEN}

Este trabajo consiste en un análisis cuantitativo del mantenimiento de las referencias anafóricas en los discursos narrativos en lengua ngäbe, en la cual pueden expresarse por medio de tres estrategias diferentes. Se muestra que los participantes absolutivos tienden a codificarse de manera léxica con mayor frecuencia que los demás, y se miden las distancias anafóricas promedio de las tres estrategias con el fin de relacionarlas con la continuidad y la accesibilidad de sus referentes.

Palabras clave: Ngäbe, guaymí, referencia, distancia anafórica, sintaxis, análisis del discurso.

\begin{abstract}
This study consists in a quantitative analysis of the maintenance of the anaphoric references in narrative discourses in Ngäbe, in which they can be expressed with three different strategies. The paper shows that the absolutive participants tend to be expressed lexically with higher frequency than the rest, and it also calculates the average anaphoric distances for the three strategies in order to relate them to their referents' continuity and accessibility.
\end{abstract}

Key Words: Ngäbe, guaymí, reference, anaphoric distance, syntax, discourse analysis. 


\section{Introducción}

\subsection{La lengua y sus hablantes}

El ngäbe (pronunciado /'yəbe/), también conocido como guaymí, es un idioma que pertenece a la familia chibchense. De acuerdo con la clasificación de Constenla (2008), se incluye en el grupo ístmico, en el que forma el subgrupo oriental junto con el cuna y el idioma genealógicamente más cercano, el bocotá. El ngäbe es la más hablada de las lenguas chibchenses, con más de 165000 usuarios (Quesada, 2007: 34-35). La gran mayoría de ellos, unos 163 000, son panameños que habitan, principalmente, en la Comarca Ngäbe-Buglé y en las provincias de Chiriquí, Veraguas y Bocas del Toro (Quesada y Vejerano, 2010: xiv). Los hablantes del lado costarricense, quienes suman alrededor de 2 500, se encuentran sobre todo en la zona fronteriza con Panamá por la costa pacífica (ibid.).

\subsection{Propósito del trabajo}

El presente trabajo consiste en un análisis cuantitativo de las referencias anafóricas en ngäbe. En esta lengua son frecuentes las anáforas cero como referencias de los participantes discursivos de la tercera persona. Dicho fenómeno se registra en contextos de alta continuidad anafórica, donde el referente es accesible para el oyente y, por lo tanto, no hace falta que se exprese por medio de un sintagma nominal. Una segunda estrategia no explícita para aludir a los participantes discursivos en ngäbe es el uso del pronombre personal de tercera persona. Con este estudio se pretende dar cuenta del grado de accesibilidad que permite que los referentes se expresen de manera atenuada, y determinar si la expresión léxica de los argumentos verbales, llevada a cabo por medio de sintagmas nominales, tiene alguna relación con el caso gramatical.

\section{La codificación de los casos gramaticales y los argumentos verbales}

La marcación de casos en ngäbe es del tipo ergativo-absolutiva. Típicamente, el participante más agentivo de las cláusulas transitivas es indicado con el caso ergativo. El marcador del ergativo es un morfema que presenta los alomorfos kägwe, kä, kwe y qwe $e^{1}$.Sin embargo, dependiendo de una serie de criterios semánticos, la ergatividad no se da en todas las construcciones transitivas (véase Quesada, (2008:160-167)). En tales circunstancias, el participante más agentivo de la cláusula aparece en caso dativo o absolutivo. El caso no marcado es el absolutivo, ya que este no recibe ningún indicador morfológico. El único argumento de las cláusulas intransitivas así como el complemento directo de las transitivas son los elementos que más típicamente se clasifican como absolutivos. Respecto al complemento indirecto, este recibe el caso gramatical de dativo, indicado por medio del morfema ie.

En lo que concierne a la posición de los marcadores de caso, se colocan detrás del sintagma al que se refieren, tal como se aprecia en el ejemplo 1 (Quesada y Vejerano, 2010: $150 ; 3)^{2}$ :

\section{(1) bobota kägwe ngwinintäri mesele ie sapo ERG preguntar-REC cangrejoDAT el sapo le preguntó al cangrejo'}

En cambio, cuando el argumento no se expresa explícitamente, los marcadores de caso aparecen detrás del verbo, como en 2 (Quesada y Vejerano 2010: 133;40):

\section{(2) Nini kwe ie \\ decir-REC ERG DAT \\ 'Le dijo'}

En ngäbe, es frecuente que el participante que funciona como sujeto pragmático, es decir, el «participante central», cumpla el papel de dativo cuando emplea el rol semántico de experimentador. De acuerdo con Quesada (2008: 142-143), esto sucede con los verbos «relativos a 
estados mentales, percepciones y sensaciones o existencia». Algunos de estos verbos expresan sus sujetos en dativo independientemente del tiempo verbal, mientras que otros lo hacen solamente cuando aparecen en tiempo indefinido. A continuación se ofrecen dos ejemplos de verbos con el participante central marcado como dativo, tomados de Quesada (2008: 142):

(3) nu tuin mä ie perro ver-IND usted DAT 'usted ve el perro'

(4) kuni ie ñö bätä hallar DAT agua hacia '[lo] halló en la ribera del río'

El ngäbe presenta el orden de constituyentes básico SOV (sujeto - objeto directo - verbal) (Quesada, 2008: 151-153), como se observa en el siguiente ejemplo (Quesada y Vejerano 2010: 175;15), donde $t i$ 'yo' es el sujeto y kura 'tigre' es el objeto directo:

ti gwe kura dägwä käräita mäie

yo ERG tigre cabeza pedir-FUT2-ITER usted-DAT

'yo le volveré a pedir la cabeza del tigre'

No obstante, por motivos pragmáticos, este orden puede ser alterado. Como ejemplo, la oración en 6 (Quesada y Vejerano, 2010: 134;76) presenta el orden OVS:

(6) Jüdrün ngö nuri kura gwe

cosa ruido tocar-REC tigre ERG

'El tigre escuchó el sonido de algo'

Los verbos en ngäbe solo presentan morfemas que marcan las categorías de tiempo-aspecto-modo y la diátesis, y carecen de morfemas de persona. Esto quiere decir que la única manera de especificar la persona gramatical de los argumentos verbales es mediante sintagmas nominales completos (para la tercera persona) o pronombres personales. Sin embargo, tal especificación es obligatoria solamente para la primera y la segunda persona, $\mathrm{y}$ es frecuente que los argumentos verbales de tercera persona no se mencionen por medio de ningún elemento nominal, es decir, que la referencia se realice mediante una anáfora cero.
En el ejemplo 2, repetido abajo como 7 (Quesada y Vejerano 2010: 133;40), se aprecia un verbo cuyos argumentos, el sujeto y el complemento indirecto (los cuales representan los participantes ergativo y dativo, respectivamente), no son expresados explícitamente:

\section{Nini kwe ie \\ decir-REC ERG DAT \\ 'Le dijo'}

En el ejemplo 8 (Quesada y Vejerano, 2010: 133;42), el participante ergativo, muria 'quinco', se detalla con un sustantivo, mientras que el dativo se manifiesta por medio de una anáfora cero:

\section{Muria gwe nini ie quinco ERG decir-REC DAT \\ 'El quinco le dijo'}

Cuando al hablante le parece necesario expresar dos argumentos explícitamente, se registran oraciones como las presentes en el siguiente ejemplo (Quesada y Vejerano, 2010: 120;17), donde tanto el participante ergativo (san 'boa') como el dativo (muria 'quinco') son representados por sustantivos:

(9) San kägwe nini muria ie boa ERG decir-REC quinco DAT 'La boa le dijo al quinco'

El ngäbe distingue morfológicamente entre el singular y el plural. El singular es el número gramatical no marcado, mientras que el plural se puede indicar por medio del morfema independiente tre, el cual se coloca atrás del elemento determinado, como se aprecia en el ejemplo 10 (Quesada y Vejerano, 2010: 129;33):

(10) jurin tre mada kägwe ja kiti biti küse biti mono PL otro ERG REF almacenar-REC sobre mano sobre

'los monos se taparon con las manos'

Sin embargo, este marcador se puede utilizar solo con seres animados, lo cual explica por qué no aparece junto con küse 'mano' en el ejemplo anterior. Además, no se emplea cuando la pluralidad es expresada mediante un numeral o en una cláusula anterior. El morfema puede aparecer aun cuando el referente no es explícito, 
por lo que la elisión de un sintagma nominal no afecta la marcación del plural; en estos casos, el marcador se coloca detrás del marcador de caso gramatical, como en el ejemplo 11 (Quesada y Vejerano, 2010: 125;3):

Köbö kidi mägä kwe tre.

plazo cerrar-REC dentro.de.cuatro.días ERG PL

'Pusieron un plazo de cuatro días.'

En el caso de argumentos absolutivos que no se expresan explícitamente, el morfema del plural se coloca detrás del verbo, como se observa en 12 (Quesada y Vejerano 2010: 140;9):

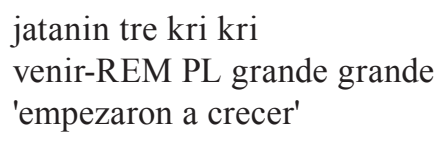

jatanin tre kri kri venir-REM PL grande grande 'empezaron a crecer'

De acuerdo con Quesada (2008: 153154), la elisión del sujeto o de los complementos se permite cuando el referente se ha expresado anteriormente y se puede inferir del contexto; por esto, el ngäbe se registra parcialmente como una lengua pro-drop en la tercera persona. Sin embargo, no se detalla acerca del asunto y, hasta el momento, no se han publicado otros trabajos sobre este fenómeno en ngäbe. Por tal razón, el presente artículo tiene como objetivo la cuantificación del mantenimiento de la referencia anafórica en esta lengua, con el fin de dar cuenta de los patrones que regulen la expresión explícita o no de los referentes discursivos.

\section{Las referencias anafóricas}

Este trabajo aborda su objeto de estudio desde la perspectiva teórica de la gramática funcional tipológica. Esta corriente parte de la premisa de que la relación entre la forma y el significado a nivel gramatical no es arbitraria, sino intencional, de tal manera que la estructura gramatical refleja el significado de manera icónica. Si se vincula este punto de vista con la codificación de las referencias anafóricas, el uso de una anáfora cero en lugar de una explícita representaría la alta accesibilidad o continuidad del referente. En contextos de baja continuidad anafórica del referente, donde el oyente puede tener problemas para comprender cuál es el referente exacto de un elemento anafórico (comúnmente porque no ha sido mencionado a lo largo de varias cláusulas), es necesario que el hablante utilice una construcción que lo exprese de manera más explícita. Esta especificación tiene como objetivo facilitarle la comprensión al oyente, pues el hablante, por supuesto, siempre está consciente de cuál es el referente.

Givón (2001a: 417) propone que, en el inglés, existen cuatro estrategias gramaticales para expresar referencias anafóricas: el cero anafórico, los pronombres anafóricos átonos, los pronombres independientes tónicos, y los sintagmas nominales completos definidos. En cambio, en ngäbe se pueden identificar tres estrategias:

\section{- cero anafórico \\ - pronombre personal \\ - $\quad$ sintagma nominal completo}

Cabe recordar que ni la primera ni la tercera estrategia pueden ser utilizadas para referirse a la primera o a la segunda persona. En 13, se ejemplifica el cero anafórico (Quesada y Vejerano, 2010: 112;1-4), donde el participante central, una persona, se introduce mediante un sintagma nominal en la primera cláusula y se expresa por medio de una anáfora cero en las dos cláusulas seguidas. Aunque se menciona la palabra muria 'quinco/guatusa' en 13a, el oyente puede deducir, dado el contexto, que el agente en $13 \mathrm{~b}$ y $13 \mathrm{c}$ no es una guatusa sino la persona.

(13)a. Ngäbe nigani muria kraire, dere ne näre, gente ir-REM quinco cazar-IND tarde esto igual

'Una persona fue a cazar guatusas;'

b. ja ketaningä kwe kä teri,

REF cercar-REM ERG espacio dentro 'se encerró en un lugar,'

c. biti nämbre kürü kiriani kä mugä te ta luego estar-REM arma insertar-REM espacio hoyo en por

'y estaba con la escopeta metida a través de una rendija' 
El ejemplo 14, tomado de Quesada y Vejerano (2010: 129;1-2), muestra el uso del pronombre personal de la tercera persona, niara. $\mathrm{Su}$ referente se introduce en $14 \mathrm{a}$ y es evidente que el pronombre, semánticamente, no aporta más información de lo que haría una anáfora cero. En este caso, parece emplear una función enfática de contraste, de la misma manera que el pronombre personal en la traducción libre al español. En 14a también se puede observar el uso de un sintagma nominal como argumento verbal. En este caso, el cuantificador togwä 'uno', parte del sintagma nominal, está separado del sustantivo que califica, ya que se coloca al otro lado del verbo.

(14)a. Jurin täni krati kä togwä te. mono estar-REC uno monte en

'Estaba una vez un mono en la montaña.'

b. Niara be kugwe kri;

él solamente palabra grande

'Solo él tiene voz fuerte;'

Como se observa en 15 (Quesada y Vejerano, 2010: 150;1-3), un referente puede volver a explicitarse mediante un sintagma nominal; esto con la finalidad de especificar el referente en casos de posible ambigüedad:

(15)a. Bobota nigui bati kä tuin, sapo ir-REC una.vez espacio ver-IND 'Cierta vez un sapo se fue de paseo,'

b. angwene ngätä mesele be; entonces reunir-REC cangrejo con 'y se encontró con un cangrejo;'

c. bobota kägwe ngwinintäri mesele ie: sapo ERG preguntar-REC cangrejo DAT 'el sapo le preguntó al cangrejo:'

De acuerdo con Givón (2001a), la lista de construcciones anafóricas representa una escala de continuidad referencial del contexto en el que se utilizan dichas estrategias. Este tipo de sucesión es denominada "escala de marcación de accesibilidad" por Ariel (1990: 73).

El cero anafórico se emplea, según Givón (2001a: 418), en contextos de continuidad referencial máxima, normalmente con su elemento correferencial en la cláusula inmediatamente precedente. Por el contrario, los sintagmas nominales se utilizan para introducir participantes nuevos en el discurso o cuando su referencia no es identificable para el oyente. Los pronombres en ngäbe ofrecen la información de la persona gramatical del referente; sin embargo, el uso del pronombre es obligatorio para la primera y la segunda persona, por lo cual un pronombre de la tercera persona y una anáfora cero ofrecen exactamente la misma cantidad de información, ya que también esta segunda estrategia implica que el referente es de tercera persona. Además, los pronombres personales, a diferencia de los sintagmas nominales completos, no aporta ninguna otra información semántica sobre el referente.

El mismo fenómeno es descrito por Chafe (1994), quien resalta la distinción entre información dada, accesible y nueva: la información dada es la que el hablante supone que el oyente tiene presente en la conciencia, por el hecho de que ya ha sido mencionada en el presente discurso y se encuentra activada. Por otro lado, la información accesible es la que el hablante supone que el oyente conoce, pero que se encuentra en un estado semiactivo. Por último, la información nueva es la que el hablante piensa que está activando en la conciencia del oyente en el momento de mencionar un referente por la primera vez. El autor (p. 75-76) afirma que la información dada parece expresarse de manera menos explícita en todas las lenguas del mundo, típicamente por medio de un pronombre átono o una anáfora cero. De la misma manera, la información nueva o accesible se suele expresar mediante sintagmas nominales completos. Chafe (1994) afirma que los sintagmas nominales completos también se emplean para expresar información dada para evitar ambigüedad en casos de dos o más posibles referentes. El número de referentes que pueden estar activos al mismo tiempo es relativamente bajo, y para que un referente siga en el estado activo, tiene que mencionarse continuamente.

Por su lado, Du Bois (1987) postula la existencia de una serie de "limitaciones de estructura argumental preferida", relacionadas con la expresión de argumentos verbales en las 
lenguas del mundo. Según su teoría, se tiende a utilizar solo un argumento léxico en cada cláusula, siendo el argumento ergativo el que menos frecuentemente se expresa de manera léxica. Esto se relaciona con el hecho de que solo se suele introducir un nuevo participante en cada cláusula y que los nuevos participantes normalmente aparecen en caso absolutivo.

Una manera de medir la continuidad anafórica es mediante la denominada "distancia anafórica": cuantas más cláusulas hay entre un elemento anafórico y la última aparición de un elemento correferencial, mayor es la distancia anafórica y menor es la continuidad. Según Givón (2001a: 463), la distancia anafórica no es una medida directa de la accesibilidad mental de los referentes, pero ofrece una buena indicación al respecto.

Cabe señalar que Quesada (2007: 67-68) afirma que el ngäbe posee un sufijo - $e$ que se agrega a los sintagmas nominales que representan participantes reintroducidos en el discurso para indicar que el sintagma tiene una función anafórica. Sin embargo, según el autor (2008: 73-74), este sufijo (que presenta el alomorfo -i) tiene un valor posesivo. Sí cumple una función anafórica ya que su antecedente es el poseedor de la entidad representada por el sintagma al que se une, pero, a juzgar por los ejemplos que presenta, su uso no parece ser restringido a los participantes reintroducidos.

Se han realizado pocos estudios acerca de las estrategias anafóricas de otras lenguas chibchenses. Como puntos de comparación para el presente estudio, lenguas como el bribri y el boruca son las más relevantes, ya que no presentan concordancia de persona en el verbo y disponen de las tres mismas estrategias anafóricas que posee el ngäbe. De acuerdo con Jara (2003), la estrategia de pronombres personales es la más común en bribri, mientras que la anáfora cero se emplea con muy baja frecuencia. Los sintagmas nominales se utilizan, principalmente, para introducir o reintroducir participantes en el discurso. Al contrario, el estudio del boruca, a cargo de Salas (1990), indica que la estrategia léxica es la más productiva en esta lengua, aunque los pronombres personales también son frecuentes. La anáfora cero en boruca, al igual que en bribri, es muy poco común. En cuanto al malecu, el cual presenta flexión para persona en el verbo, Krohn (2012) muestra que la estrategia menos explícita, la flexión verbal sin ningún sintagma nominal o pronombre correferencial, es la más frecuente y se emplea con una distancia anafórica promedio de alrededor de 2 cláusulas. La construcción que incluye un pronombre personal, la cual no representa un método semánticamente más explícito en la mayoría de los casos, es poco frecuente y se utiliza con una distancia anafórica de hacia 3,5 cláusulas en promedio. Por último, la estrategia más explícita en malecu, la de sintagma nominal completo correferencial con la flexión verbal, presenta una distancia anafórica promedio de más de 4,8 cláusulas.

\section{Metodología}

\subsection{El corpus analizado}

El corpus consiste en 18 narraciones ngäbes publicadas en el libro Kugwe ngäbere. Leyendas y tradiciones ngäbes por Quesada y Vejerano (2010). Dicho libro contiene un total de 37 textos, de los cuales los primeros 18, según los autores, «están en estrecha relación con el mundo mítico guaymí» e incluyen mitos y leyendas tradicionales (Quesada y Vejerano, 2010: xviii). Las siguientes 18 narraciones

tienen que ver con relatos humorísticos, cuentos de animales personificados, donde resalta la astucia del quinco o guatusa (Dasyprocta punctata), junto con episodios anecdóticos que sucedieron en algún momento, tanto lejano como cercano. (Quesada y Vejerano, 2010: xix)

Por último, el libro también incluye un canto. Para la presente investigación se decidió trabajar con los 18 relatos humorísticos y de animales personificados, ya que estos tienden a incluir una variedad de personajes más limitada que los mitos y las leyendas. Además, los personajes suelen ser más recurrentes a lo largo de los textos, por lo que estos escritos 
son más aptos para análisis cuantitativos del mantenimiento de la referencia anafórica.
Los 18 textos examinados en este trabajo se exponen en la Tabla 1, con sus títulos en ngäbe y en español:

TABLA 1

Textos incluidos en el corpus

\begin{tabular}{lll}
\hline $\mathbf{N}^{\mathbf{0}}$ & Título en ngäbe & Título en español \\
\hline 1 & Ni grö mogre & El hombrecillo celoso \\
\hline 2 & Muria kraigä & El cazador de guatusas \\
\hline 3 & Jurin uai & La imagen del mono \\
\hline 4 & Ngöbö bätä muria & Dios y el quinco \\
\hline 6 & Bura bätä bobota & El venado y el sapo \\
\hline 7 & Jurin bätä usulin & El mono y el trueno \\
\hline 8 & Kura bura & El tigre y el venado \\
\hline 9 & Ngüsülü kura & El grillo y el tigre \\
\hline 10 & Mesele bobota & El cangrejo y el sapo \\
\hline 11 & Muria bätä laga & El quinco y el lagarto \\
\hline 12 & Muria bätä meri & El quinco y la mujer \\
\hline 13 & Muria mesele & El quinco y el cangrejo \\
\hline 14 & Muria sulia & El quinco y el hispano \\
\hline 15 & $\begin{array}{l}\text { Muria, gwa kri, } \\
\text { mölö }\end{array}$ & El quinco, la ballena y la danta \\
\hline 16 & $\begin{array}{l}\text { Muria, kura bätä } \\
\text { bura }\end{array}$ & El quinco, el tigre y el venado \\
\hline 17 & Ni kri bätä bobota & El gigante y el sapo \\
\hline 18 & Sulia rä burere & El hispano que hedía a saíno \\
\hline
\end{tabular}

En Quesada y Vejerano (2010), los textos están segmentados en partes enumeradas, las cuales, en algunas ocasiones, corresponden a cláusulas. Las referencias de los ejemplos tomados del corpus incluyen el número de la página en la que se encuentran en el libro original y el número del segmento, separados por punto y coma. Por ejemplo, Quesada y Vejerano (2010: 117;15) significa segmento número 15 de la página 117 del corpus. Similarmente, Quesada y Vejerano (2010: 110;8-9) se refiere a los segmentos 8-9 del relato de la página 110 del libro de Quesada y Vejerano.

En el libro fuente, cada texto aparece en tres versiones: la versión original en ngäbe, traducido literalmente morfema por morfema, y en una traducción libre al español. En el presente artículo se utilizan las tres versiones ${ }^{3}$.Sin embargo, en algunos casos, la traducción libre al español se ha modificado ligeramente, con el fin de que los ejemplos resulten más claros. 


\subsection{La división de los textos en cláusulas}

Los análisis requerían que los textos se dividieran en cláusulas. Una cláusula es definida como "la expresión lingüística de una proposición" por Payne (1997), mientras que Givón (1990) describe la proposición como "la unidad básica del almacenamiento de información”. En el proceso de división del texto en cláusulas, se aplicó como regla general que cada verbo junto con sus argumentos y los otros elementos relacionados con el evento/estado expresado por el verbo forman una cláusula. El ngäbe presenta una cantidad considerable de composiciones verbales, es decir, dos o más verbos diferentes que funcionan como una unidad semántica y que, por lo tanto, deben ser tratados como parte de la misma cláusula. En varios casos resulta difícil determinar si los verbos deben considerarse una composición verbal o no, sin embargo, la clasificación se intentó llevar a cabo de manera consistente. En el ejemplo 16 (Quesada y Vejerano, 2010: 116;1) se nota claramente que los dos verbos, nämbre 'estaba' y kraire 'acechaba', forman parte de una sola proposición, la cual alude a la acción de cazar/acechar:

(16) Ni nämbre bura kraire persona estar-REM venado cazar-IND

'Estaba un hombre acechando venados'

También la construcción que se aprecia en 17 (Quesada y Vejerano, 2010: 116;10), la cual utiliza tres verbos seguidos, se consideró una sola cláusula porque, en realidad, se trata de una sola acción:

(17)

jurin niberaningä nen kitani

mono colgar-REM correr-IND venirREM

'el mono salió corriendo'

De manera similar, los dos verbos en el siguiente ejemplo (Quesada y Vejerano, 2010: 133:41) forman una unidad semántica:

(18) Matare ti bigue mä kwete hoy yo intentar-IND usted comer-IND 'Hoy voy a intentar comérmelo'
Las decisiones tomadas con respecto a las composiciones verbales son importantes, dado que influyen en los resultados de los análisis cuantitativos. Por ejemplo, si la construcción en 16 fuera clasificada como dos cláusulas, habría que postular que la primera es intransitiva y que su único argumento es léxico, mientras que la segunda sería transitiva con un argumento ergativo elidido y un argumento absolutivo léxico. En cambio, al considerar que existe solo una proposición en el enunciado, se trataría de una sola cláusula transitiva con dos argumentos léxicos. Similarmente, si el ejemplo 17 se considerara como tres cláusulas, dos de ellas no contarían con argumento léxico, lo cual incrementaría el total de participantes absoulutivos no léxicos. Además, el segmentar las composiciones verbales en varias cláusulas, influiría en los resultados de la medición de las distancias anafóricas promedio.

En contraste, en el ejemplo 19 (Quesada y Vejerano 2010: 125;4), los dos verbos ürügrö 'recogió' y miri 'puso' deben considerarse independientes, aunque el segundo aparezca inmediatamente después del primero; esto porque hacen referencia a dos acciones seguidas y, por lo tanto, constituyen dos cláusulas diferentes:

(19)a. Käne bobota gwe bobota mada ürügrö primeramente sapo ERG sapo otro recoger-REC

'Primeramente el sapo recogió a otros sapos,'

b. miri kwe ja jie biti, poner-REC ERG REF camino-DAT sobre 'los puso en fila,'

También aparecen cláusulas sin ninguna forma verbal; siempre que expresen una proposición, cumplen con la definición de cláusula. Estas suelen traducirse con un verbo copulativo en español, como se observa en 20 (Quesada y Vejerano, 2010: 110;3):

(20) Ni ñagare ya? persona no INTERR '¿No ha estado nadie aquí?' 


\subsection{Procedimiento del análisis}

En primer lugar, se buscó comprobar las "limitaciones de estructura argumental preferida" de Du Bois, donde en autor afirma que se tiende a evitar la expresión léxica del argumento absolutivo. Por lo tanto, se llevó a cabo un conteo del tanto por ciento de los argumentos de cada caso gramatical (ergativo, absolutivo y dativo) que se mencionan de manera léxica en el corpus con el fin de constatar si, efectivamente, son los referentes absolutivos los más frecuentemente expresados mediante un sintagma nominal. Cabe señalar que el estudio se realizó siguiendo estrictamente la marcación morfológica de casos. Por lo tanto, un referente que, dado el carácter del ngäbe como una lengua ergativa restringida, no se marca como ergativo en ciertas cláusulas transitivas -aunque claramente es el participante más agentivotampoco se registró como ergativo, sino como absolutivo o dativo según correspondiera. Todas las cláusulas que incluyen un verbo, tanto las intransitivas como las transitivas, deben contener un participante absolutivo. Por ello, si no aparece ningún argumento sin marcador de caso, se considera que esa cláusula tiene un participante absolutivo no léxico. Esto se ejemplifica en el 21 (Quesada y Vejerano, 2010: 145;4), donde lo que fue construido (una casa) constituye el argumento absolutivo.

\section{(21) Siribeninta kwe bobota}

hacer-REC-ITER ERG otra.vez

'Por segunda vez la construyó'

La única excepción a la última regla fue con respecto al tema verbal ñe- 'decir'. Su complemento directo siempre es una cláusula, no solamente un sintagma nominal referido a un participante discursivo, por lo que no se consideró relevante tomar en cuenta estos argumentos en un análisis de las referencias anafóricas. En cambio, los argumentos ergativos y dativos se registraron solamente en las cláusulas donde aparecen los marcadores morfológicos de estos casos. Por lo tanto, en una cláusula que contiene un verbo que típicamente presenta un participante en caso dativo, no se registró ningún argumento dativo si no había presencia del marcador de dativo, ie.

En segundo lugar, se realizó un análisis de la distancia anafórica promedio de las tres estrategias anafóricas del ngäbe -anáfora cero, pronombre personal y sintagma nominal completo- siguiendo el procedimiento de Givón (1983, 2001a): Para cada referencia de un participante, explícito o no, se contó el número de cláusulas desde la última aparición del mismo referente. Se empleó un máximo arbitrario de 20, ya que, de acuerdo con Givón (1983: 443), el oyente normalmente no es capaz de identificar un referente a mayor distancia. Es importante señalar que las referencias de participantes que se introducen por primera vez en el discurso no son anafóricas, por lo que no se les asignó ninguna distancia anafórica.

En 22 (Quesada y Vejerano 2010: 175;1-4) se presentan las seis primeras cláusulas del texto 16 del corpus, las cuales ilustran cómo se llevó a cabo el análisis de las distancias anafóricas promedio:

(22)a. Kä togwä te muria nämä. espacio espina en quinco estar-PT 'Caminaba un quinco en la montaña.'

b. Ju nämbre bängarabe kä togwä te jutuani muria ie, casa estar-REM hermoso espacio espina en encontrar-REM quinco DAT

'El quinco encontró una hermosa guarida en medio de la montaña,'

c. kura aibe nüne ju ne te. tigre solamente vivir-IND casa esto en 'en la cual vivían tigres.'

d. Muria tönibi ju ne diaingä kura tre kän, quinco querer-IND casa esto apoderarseFUT2 tigre PL de 'El quinco quiso apoderarse de esa guarida de tigres,'

e. nigui töibigare ir-REC pensar-FIN 'y se fue pensando'

f. ñö ju ne rabare kwe. cómo casa esto quedar-FIN2 ERG 'cómo apoderarse de ella.' 
En la cláusula 22a se introducen dos participantes: kä togwä 'montaña' y muria 'quinco'; como se trata de su primera aparición en el discurso, no se consideran referencias anafóricas. Por su parte, en 22b, ambos participantes se vuelven a mencionar mediante sintagmas nominales, por lo que se anotan dos ocurrencias de referencias anafóricas léxicas con la distancia de una cláusula. Además, se introduce un nuevo participante, $j u$ 'casa'. En 22c, la cual es subordinada a $22 \mathrm{~b}$, vuelve a mencionarse $j u$ 'casa', y se le asigna otra referencia de distancia 1 a la estrategia de sintagma nominal. Kura 'tigre' también se introduce en el discurso en este punto. En 22d hay una nueva referencia de muria 'quinco'. Esta vez, se anota una referencia con la distancia de dos cláusulas para la estrategia de sintagma nominal, ya que la última vez que se mencionó el mismo participante fue dos cláusulas antes, en 22b. Además, ju 'casa' y kura 'tigre' representan otras dos referencias a una unidad de distancia. 22e contiene una anáfora cero que se refiere al quinco, mencionado en la cláusula anterior. Por último, en $22 \mathrm{f}$ hay una nueva referencia implícita del tigre, lo cual constituye otra anáfora de distancia 1 , y una referencia de $j u$ 'casa', mencionada en $22 \mathrm{~d}$, del tipo léxico; a esta se le asigna una distancia de dos cláusulas.

\section{Resultados del análisis}

\subsection{La expresión léxica de los argumentos verbales}

Los resultados de la primera sección del análisis, el número de argumentos léxicos de cada caso gramatical, se presentan en la Tabla 2. Cada fila representa un texto del corpus, cuyo número (cf. la Tabla 1) se indica en la primera columna. Hay dos columnas para cada uno de los casos gramaticales ergativo, absolutivo y dativo: la primera indica el número de apariciones léxicas, entre el número total de aparaciones del caso en cuestión, mientras que la segunda columna muestra el porcentaje de expresiones léxicas de cada caso gramatical.

TABLA 2.

Referencias léxicas de cada caso gramatical

\begin{tabular}{lllllll}
\hline Texto & \multicolumn{2}{c}{ Ergativo } & \multicolumn{2}{c}{ Absolutivo } & \multicolumn{2}{c}{ Dativo } \\
\hline 1 & $1 / 4$ & $25,0 \%$ & $7 / 12$ & $58,3 \%$ & $2 / 5$ & $40,0 \%$ \\
\hline 2 & $2 / 11$ & $18,2 \%$ & $33 / 45$ & $73,3 \%$ & $2 / 7$ & $28,6 \%$ \\
\hline 3 & $4 / 7$ & $57,1 \%$ & $12 / 27$ & $44,4 \%$ & $1 / 4$ & $25,0 \%$ \\
\hline 4 & $13 / 22$ & $59,1 \%$ & $24 / 38$ & $63,2 \%$ & $7 / 16$ & $43,8 \%$ \\
\hline 5 & $5 / 5$ & $100 \%$ & $16 / 19$ & $84,2 \%$ & $2 / 6$ & $33,3 \%$ \\
\hline 6 & $9 / 10$ & $90,0 \%$ & $27 / 33$ & $81,8 \%$ & $5 / 9$ & $55,6 \%$ \\
\hline 7 & $4 / 24$ & $16,7 \%$ & $52 / 83$ & $62,7 \%$ & $4 / 23$ & $17,4 \%$ \\
\hline 8 & $3 / 14$ & $21,4 \%$ & $31 / 51$ & $60,8 \%$ & $4 / 10$ & $40,0 \%$ \\
\hline 9 & $9 / 16$ & $56,3 \%$ & $28 / 40$ & $70,0 \%$ & $4 / 7$ & $57,1 \%$ \\
\hline 10 & $8 / 11$ & $72,7 \%$ & $15 / 24$ & $62,5 \%$ & $4 / 11$ & $36,4 \%$ \\
\hline 11 & $6 / 13$ & $46,2 \%$ & $19 / 33$ & $57,6 \%$ & $8 / 11$ & $72,7 \%$ \\
\hline 12 & $8 / 20$ & $40,0 \%$ & $27 / 49$ & $55,1 \%$ & $13 / 18$ & $72,2 \%$ \\
\hline 13 & $1 / 7$ & $14,3 \%$ & $22 / 30$ & $73,3 \%$ & $4 / 5$ & $80,0 \%$ \\
\hline 14 & $10 / 20$ & $50,0 \%$ & $58 / 82$ & $70,7 \%$ & $12 / 22$ & $54,5 \%$ \\
\hline 15 & $3 / 9$ & $33,3 \%$ & $25 / 26$ & $96,2 \%$ & $3 / 9$ & $33,3 \%$ \\
\hline 16 & $11 / 27$ & $40,7 \%$ & $72 / 106$ & $67,9 \%$ & $8 / 23$ & $34,8 \%$ \\
\hline 17 & $4 / 4$ & $100 \%$ & $18 / 22$ & $81,8 \%$ & $4 / 6$ & $66,7 \%$ \\
\hline 18 & $5 / 12$ & $41,7 \%$ & $17 / 23$ & $73,9 \%$ & $0 / 0$ & - \\
\hline Total & $\mathbf{1 0 6 / 2 3 6}$ & $\mathbf{4 4 , 9 \%}$ & $\mathbf{5 0 3 / 7 4 3}$ & $\mathbf{6 7 , 7 \%}$ & $\mathbf{8 7 / 1 9 2}$ & $\mathbf{4 5 , 3 \%}$ \\
\hline & & & & & & \\
\hline
\end{tabular}


Primeramente, se revela un patrón muy ostensible en relación a los participantes absolutivos: En todos los textos, con excepción del número 3, más de la mitad de las referencias son léxicas. El número más alto aparece en el texto 15 , donde 25 de las 26 referencias de participantes absolutivos son léxicas. Sumando todos los textos del corpus, son léxicas un $67,7 \%$ de las ocurrencias del caso absolutivo.

El segundo aspecto que se aprecia es el hecho de que las referencias de los casos ergativo y dativo son de un carácter más variado, dado que las menciones léxicas predominan en algunos textos, y las no léxicas en otros. Por ejemplo, la totalidad de las referencias del ergativo en los textos 5 y 17 son sintagmas nominales, mientras que, en el texto 13, solo uno de siete argumentos con este caso gramatical es léxico.
En todo el corpus, las referencias léxicas del ergativo y el dativo presentan aproximadamente la misma frecuencia: $44,9 \%$ para el ergativo y $45,3 \%$ para el dativo; es decir, un poco menos de la mitad de las refencias, un porcentaje considerablemente menor que las menciones léxicas del caso absolutivo.

\subsection{Las distancias anafóricas promedio}

La segunda parte del análisis consistió en calcular la distancia anafórica promedio de las tres estrategias anafóricas en ngäbe. La Tabla 3 muestra el promedio de cada estrategia en cada texto del corpus. Entre paréntesis se anota el número de referencias anafóricas de los tres tipos en el texto en cuestión.

TABLA 3.

Distancia anafórica promedio de cada estrategia en cada texto del corpus y número total de apariciones de la estrategia entre paréntesis

\begin{tabular}{llllllllll}
\hline & $\mathbf{1}$ & $\mathbf{2}$ & $\mathbf{3}$ & $\mathbf{4}$ & $\mathbf{5}$ & $\mathbf{6}$ & $\mathbf{7}$ & $\mathbf{8}$ & $\mathbf{9}$ \\
\hline Anáfora cero & 2,00 & 1,41 & 1,13 & 1,16 & 1,10 & 1,00 & 2,13 & 1,33 & 1,29 \\
& $(10)$ & $(34)$ & $(23)$ & $(25)$ & $(7)$ & $(12)$ & $(58)$ & $(51)$ & $(14)$ \\
\hline Pronombre personal & - & 1,00 & - & - & - & 1,67 & - & 1,00 & 1,00 \\
& $(0)$ & $(1)$ & $(0)$ & $(0)$ & $(0)$ & $(3)$ & $(0)$ & $(1)$ & $(1)$ \\
\hline Sintagma nominal & 2,67 & 3,45 & 3,33 & 2,98 & 3,40 & 2,93 & 2,85 & 2,67 & 3,19 \\
& $(12)$ & $(22)$ & $(9)$ & $(42)$ & $(17)$ & $(26)$ & $(39)$ & $(33)$ & $(42)$ \\
\hline
\end{tabular}

\begin{tabular}{lllllllllll}
\hline & $\mathbf{1 0}$ & $\mathbf{1 1}$ & $\mathbf{1 2}$ & $\mathbf{1 3}$ & $\mathbf{1 4}$ & $\mathbf{1 5}$ & $\mathbf{1 6}$ & $\mathbf{1 7}$ & $\mathbf{1 8}$ & Total \\
\hline Anáfora cero & 1,38 & 1,75 & 1,29 & 1,14 & 1,50 & 1,38 & 1,36 & 1,25 & 1,11 & $\mathbf{1 , 4 5}$ \\
& $(13)$ & $(20)$ & $(31)$ & $(21)$ & $(24)$ & $(8)$ & $(42)$ & $(4)$ & $(9)$ & $\mathbf{( 4 0 6 )}$ \\
\hline Pronombre personal & - & - & 1,00 & - & 2,00 & - & 2,67 & 1,00 & - & $\mathbf{1 , 7 4}$ \\
& $(0)$ & $(0)$ & $(2)$ & $(0)$ & $(2)$ & $(0)$ & $(3)$ & $(1)$ & $(0)$ & $(\mathbf{1 4})$ \\
\hline Sintagma nominal & 1,88 & 3,00 & 3,81 & 2,63 & 4,08 & 3,79 & 3,23 & 2,48 & 3,14 & $\mathbf{3 , 1 9}$ \\
& $(25)$ & $(27)$ & $(48)$ & $(24)$ & $(60)$ & $(33)$ & $(81)$ & $(23)$ & $(14)$ & $\mathbf{( 5 7 4 )}$ \\
\hline
\end{tabular}

En lo que concierne a la anáfora cero, se observa que su distancia no excede las dos cláusulas en ningún texto; esta es de 2,00 en el texto 1 , y el promedio más bajo, 1,00 , se expresa en el texto 6. En este último, las 12 referencias de este tipo presentan una distancia de una sola cláusula. La distancia promedio de la estrategia menos explícita en todo el corpus es de 1,45 cláusulas.

Los pronombres personales son escasos; en ningún texto aparecen más de tres pronombres, y en varios relatos no hay ninguno. Además, su distancia anafórica promedio varía considerablemente entre un texto y otro. 
La distancia promedio de los 14 pronombres personales detectados en todo el corpus es de 1,74 cláusulas. Sin embargo, ya que esta cifra se basa en escasas observaciones, no resulta un dato de gran relevancia.

Por último, los sintagmas nominales completos se emplean con una distancia anafórica claramente mayor que las otras dos estrategias. En todos los textos el promedio es mayor a dos cláusulas, salvo en el caso 10, donde es de 1,88. Por lo general, se encuentra entre alrededor de 2,5 y 4 cláusulas. En todo el conjunto de narraciones, esta estrategia presenta una distancia anafórica promedio de 3,19. Además, se observa que los sintagmas nominales son usados con mayor frecuencia que las anáforas cero, con 574 ocurrencias registradas frente a las 406 de la anáfora cero.

\section{Conclusiones}

En la primera parte del análisis se confirmó que el ngäbe se ajusta a la tendencia descrita por Du Bois (1987) de que los argumentos absolutivos se expresen de manera léxica con mayor frecuencia que los demás. Mientras que los absolutivos son léxicos en aproximadamente $2 / 3$ de sus apariciones, los argumentos ergativos $\mathrm{y}$ dativos son expresados mediante esta estrategia en menos de la mitad de los casos. No obstante, esta última frecuencia también es relativamente alta; es probable que se deba a que las únicas alternativas de una estrategia léxica sean las anáforas cero y los pronombres. Estas dos opciones requieren de un referente altamente accesible, ya que, en ngäbe, estas no codifican ningún tipo de clase nominal u otro rasgo que pueda ayudar al oyente a distinguir el referente de entre otros posibles. Por ejemplo, el pronombre de la tercera persona la en español contiene el rasgo [+femenino], por lo cual el conjunto de posibles referentes es más limitado que el existente para el pronombre ngäbe niara, pues este solo expresa el rasgo de la tercera persona. Por lo tanto, es más probable que la expresión atenuada de un referente produzca más ambigüedad en ngäbe que en aquellas lenguas cuyos pronombres u otros elementos anafóricos atenuados codifican diferentes clases nominales.

En cuanto a las distancias anafóricas promedio, los resultados fueron los esperados, pues la estrategia de sintagma nominal muestra una distancia notablemente mayor que la de anáfora cero. Debido a la considerable probabilidad de ambigüedad implicada por el uso de un pronombre o una anáfora cero, tales estrategias requieren de alta continuidad del referente. Las pocas apariciones del pronombre personal de la tercera persona no son suficientes para llegar a conclusiones precisas, pero el hecho de que su distancia anafórica promedio se aproxime a aquella de la anáfora cero, concuerda con la observación de que las dos estrategias contienen exactamente la misma cantidad de información: solamente la persona gramatical. A primera vista, la función del pronombre personal parece ser enfática, como en español, pero las diferencias pragmáticas entre el pronombre de la tercera persona y la elipsis deben estudiarse con más detalle.

Si se compara el uso de las tres estrategias anafóricas en ngäbe con las correspondientes en bribri y boruca, lenguas del mismo grupo genealógico ístmico que disponen de las mismas tres estrategias y cuya morfología verbal tampoco presenta concordancia con los argumentos, destacan dos observaciones: En primer lugar, los pronombres personales en ngäbe son muy poco comunes en comparación con las otras dos lenguas. En bribri, estos constituyen la estrategia anafórica más frecuente, y también se registra un uso alto en boruca. En segundo lugar, la anáfora cero se utiliza mucho más en ngäbe que en las otras lenguas. Estos hechos indican que la tendencia de la elisión del pronombre de la tercera persona del ngäbe está mucho más avanzada que en bribri y boruca, por lo cual el uso de los pronombres personales en las dos últimas lenguas corresponderían, en gran medida, a la elisión en ngäbe; pese a todo, los pronombres no aportan más información sobre el referente que la anáfora cero. En tal caso, la función enfática que parecen poseer los pronombres en ngäbe, se llevaría a cabo de una manera distinta en bribri y boruca. De todos 
modos, estos hechos requieren de un estudio comparativo para comprenderse con detalle. El sistema de las referencias anafóricas del ngäbe se asemeja mucho más al del malecu, donde los pronombres también son poco frecuentes y parecen funcionar principalmente para expresar énfasis o contraste. Resalta el hecho de que las distancias anafóricas promedio, tanto de la elisión como de los sintagmas nominales, son menores en ngäbe que en malecu. Sin embargo, esto podría explicarse por diferencias en cuanto al número de participantes discursivos presentes y la naturaleza de los textos analizados: Entre más participantes existan en un discurso, menor continuidad anafórica tendrá cada uno, y mayor será la necesidad de especificar los referentes de manera léxica.

Como el presente análisis es de carácter cuantitativo, solamente acompañado por ciertas observaciones, todos los fenómenos deben analizarse de manera cualitativa para comprobarse y describirse con mayor detalle. Además, para entender un sistema tan complejo como es el del mantenimiento de la referencia anafórica, es esencial analizar también otros tipos de discurso, sobre todo el conversacional, el cual puede distinguirse significativamente del discurso narrativo.

\section{Notas}

1. Se remite a Quesada (2008: 155-160) para una reseña detallada de la distribución de los alomorfos.

2. En las citas textuales, las referencias que incluyen punto y coma después del número de página y un número adicional, están señalando la línea o renglón de ubicación en el texto original.

3. En este artículo se hace uso de la misma ortografía ngäbe que la utilizada por Quesada (2008) y Quesada y Vejerano (2010).

\section{Bibliografía}

Ariel, Mira. 1990. Accessing noun phrase antecedents. Nueva York: Routeledge.
Chafe, Wallace. 1994. Discourse, consciousness, and time: The flow and displacement of conscious experience in speaking and writing. Chicago: The University of Chicago Press.

Constenla Umaña, Afolfo. 2008. "Estado actual de la subclasificación de las lenguas chibchenses y de la reconstrucción fonológica y gramatical del protochibchense". Estudios de lingüística Chibcha. 27: 117-135.

Du Bois, John W. 1987. "The discourse basis of ergativity". Language. 64: 805-855.

Givón, Talmy. 1983. Topic continuity in discourse: A quantitative cross language study. Amsterdam: John Benjamins.

2001a. Syntax. Vol. I. Amsterdam: John Benjamins.

2001b. Syntax. Vol. II. Amsterdam: John Benjamins.

Jara Murillo, Carla Victoria. 2003. "Codificación de participantes en una narración bribri". Estudios de Lingüistica Chibcha. 22: 33-60.

Krohn, Haakon Stensrud. 2012. "El mantenimiento de la referencia anafórica en el discurso narrativo tradicional en lengua malecu". Revista de Filología y Lingüistica de la Universidad de Costa Rica. 38 (1): 191-216.

Payne, Thomas. 1997. Describing morphosyntax. Cambridge: Cambridge University Press.

Quesada Pacheco, Juan Diego. 2007. The Chibchan languages. Cartago: Editorial Tecnológica de Costa Rica. 
Quesada Pacheco, Miguel Ángel. 2008. DAT: posposición de dativo

Gramática de la lengua guaymí (ngäbe). Munich: Lincom Europa.

Quesada Pacheco, Miguel Ángel y Bertilo Vejerano Palacio. 2010. Kugwe ngäbere. Leyendas y tradiciones ngäbes. San Pedro: Editorial de la Universidad de Costa Rica.

Salas, Álvaro. 1990. Análisis del discurso de la narrativa boruca. Tesis de maestría: Universidad de Costa Rica.

\section{Anexo 1. Abreviaturas}

ERG: posposición de ergativo

FIN: supino o marcador de finalidad

FUT2: futuro lejano

IND: tiempo indefinido o ilimitado realis

INTERR: partícula interrogativa

ITER: marcador de iteratividad

PL: marcador de pluralidad

Las abreviaturas utilizadas en el presente trabajo son las mismas presentes en Quesada y Vejerano (2010).

- $\quad$ Linde de morfema

Separa significados o elementos del español que en conjunto traducen un solo elemento ngäbe

PT: pasado testimonial

REC: pasado reciente

REF: marcador de reflexividad o reciprocidad

REM: pasado remoto

\section{(2) $(\oplus \Theta \Theta$}

Este obra está bajo una licencia de Creative Commons Reconocimiento-NoComercial-SinObraDerivada 4.0 Internacional. 